\title{
A Bayesian Demand-Side Management Strategy for Smart Micro-Grid
}

\author{
Matteo Sola $^{1}$ - Giorgio Matteo Vitetta ${ }^{1}$
}

Received: 11 November 2015 / Accepted: 26 June 2016 / Published online: 6 July 2016

(C) Springer Science+Business Media Singapore 2016

\begin{abstract}
In this manuscript a novel strategy for distributed and autonomous demand-side energy management among users of a low-voltage micro-grid is developed. Its derivation is based on: a) modelling the energy consumption scheduling of the shiftable loads that belong to a given user as a noncooperative two-player game of incomplete information, in which the user itself plays against an opponent collecting all the other users of the same micro-grid; b) assuming that each user is endowed with statistical information about its behavior and that of its opponent, so that it can choose actions maximising its expected utility. Numerical results evidence the efficacy of the proposed strategy when employed to manage the charging of electric vehicles in a micro-grid.
\end{abstract}

Keywords Demand-side management · Game theory · Distributed algorithm · Smart grid · Plug-in hybrid electric vehicle

\section{Introduction}

In recent years a number of research activities in the field of optimization of smart power grids have addressed the

Matteo Sola

matteo.sola@unimore.it

Giorgio Matteo Vitetta

giorgio.vitetta@unimore.it

1 Department of Engineering "Enzo Ferrari”, University of Modena and Reggio Emilia,

Via P. Vivarelli 10, Modena, MO, Italy problem of demand response (also known as demand side management, DSM) and, in particular, the development of new adaptation strategies that allow to match the load demand of multiple residential end users to the power supply provided by a single load-serving entity [23]. Generally speaking, demand response in a multiuser scenario can be achieved by a) turning currently static consumers into active consumers and producers (briefly, prosumers) able to interact with an electric utility (providing time-varying energy-pricing) in real time and b) scheduling the shiftable appliances owned by such prosumers in a way that they are activated in off-peak hours, so that the peak-to-average ratio (PAR) in load demand is mitigated and the total energy cost to the utility company is reduced. In developing novel techniques for multiuser demand response, significant attention has been paid to distributed strategies [6], since they allow each prosumer to plan, on the basis of some information (concerning energy pricing or the total hourly usage of other prosumers), its load scheduling in an autonomous fashion, so that message exchanges between the load serving entity and the prosumer, or among the users are minimised. Note that the availability of some form of information sharing among prosumers is fundamental to coordinate their scheduling actions. However, this need raises the problems of a) developing a two-way data communication network interconnecting different users and the utility, and b) preserving prosumer privacy. The last issue is particularly critical, since it is expected that prosumers are unwilling to provide updated information about their real-time or daily energy consumption.

In the technical literature various algorithmic approaches have been proposed to solve the load scheduling problem in a multiuser scenario (e.g., see Giannakis et al. ([6], p. 122) and references therein); one of these approaches is based on a fundamental theoretical tool, known as game theory [16]. 
In particular, the problems of formulating energy consumption scheduling as a multi-player game ${ }^{1}$ and of developing autonomous and distributed DSM strategies minimizing the energy costs have been investigated in Mohsenian-Rad et al. [11]; Atzeni et al. [1]; Ramachandran et al. [14]. Note, however, that, on the one hand, the cooperative and non cooperative strategies proposed in Mohsenian-Rad et al. [11] and Atzeni et al. [1] rely on the assumption that a complete knowledge about the users' daily needs is available, so that DSM can be formulated as a day-ahead optimization process; on the other hand, the solution proposed in [14] is based on the adoption of an auction mechanism taking place in a stochastic energy market.

In this manuscript the problem of multiuser load scheduling in a smart micro-grid (MG) scenario [3] is tackled and a novel DSM strategy for load scheduling is developed resorting to Bayesian game theory. The proposed strategy is based on a) modelling each prosumer as a player in a repeated two-player game of incomplete information; b) adopting a simple virtual pricing model for the power exchanged by each prosumer with the MG; c) employing a mixed strategy for payoff maximization. As far as points a) and b) are concerned, it is also worth mentioning that, unlike Mohsenian-Rad et al. [11]; Atzeni et al. [1]; Ramachandran et al. [14], the following assumptions are made in our game model:

1. Each player does not know the energy scheduling or the instant (or daily) energy consumption of all the other prosumers of the same MG, but is only endowed with statistical information about its and their future overall consumptions, so that it can select its actions on the basis of an expected payoff (i.e., a Bayesian approach is adopted). Such statistical information about the overall power flow in the MG are periodically broadcasted by a MG supervisor which generates them by processing energy consumption data provided by the MG users with a fixed delay. This approach allows each prosumer to operate in an autonomous fashion and to protect its own privacy.

2. The proposed virtual pricing model refers to the power flow between the MG and each of its prosumers. Our focus on the power flow (instead of the energy flow occurring in time slots of $1 \mathrm{~h}[1,11])$ is mainly motivated by the highly dynamic nature of the energy supply from renewable sources usually available in a MG.

It is important to point out that the application of Bayesian game theory to the DSM problem in smart grids has been

\footnotetext{
${ }^{1}$ Applications of game theory to other optimization problems in smart grid can be found in Saad et al. [15]; Pedrasa et al. [12]; Fouda et al. [4].
}

first suggested by Saad et al. ([16], p. 99), where, however, no specific hint has been given for the development of novel strategies based on this approach. As far as we know, our manuscript represents the first contribution to that new research area.

The remaining part of the manuscript is organized as follows. The MG model considered in our work is described in "Micro-Grid Model". A novel DSM strategy based on game theory is developed in "Demand-Side Management Based on Bayesian Game Theory". Some performance results are illustrated in "Numerical Results", where its use in the management of the recharge of plug-in electric vehicles (PHEVs) in a MG $[19,22]$ is analysed.

\section{Micro-Grid Model}

In this manuscript we consider a low-voltage residential smart MG, composed of $N$ prosumers, i.e. distributed energy producers sharing their resources and dealing with an energy utility as a single entity (see Fig. 1). Our MG model has the following relevant features:

- Each prosumer owns the following two types of power loads: a) unshiftable loads (ULs) which can be turned on at arbitrary instants of any day; b) shiftable (i.e., controllable) loads $^{2}$ (SLs), whose activation can be flexibly managed by properly scheduling them within a specified time interval.

- Each prosumer is equipped with: a) distributed energy resources (renewable energy sources, RESs, in the considered scenario); b) a power and data management unit (called energy gateway, EG), which interfaces the prosumer itself with the MG power and neighbourhood data network (NAN), controlling local energy sources and managing some SLs through a home data network (HAN). Moreover, the $n$-th prosumer's EG (with $n=$ $1,2, \ldots, N)$ provides real time monitoring of the power $p_{n}(t)$ exchanged by the prosumer itself at time $t$ with the MG; this quantity is positive (negative) if the power is absorbed from (supplied to) the MG and satisfies the inequality

$P_{g, \text { max }}^{(n)}<p_{n}(t)<P_{a, \max }^{(n)}$

that holds at an arbitrary instant $t$; here $P_{a, \max }^{(n)}\left(P_{g, \max }^{(n)}\right)$ represents the maximum absorbed (generated) power by the $n$-th prosumer. It is important to point out that the power $p_{n}(t)$ can be expressed as

$$
p_{n}(t)=p_{n}^{(r)}(t)+p_{n}^{(s)}(t),
$$

\footnotetext{
${ }^{2}$ The specific case of PHEVs to be periodically recharged at prosumer premises is considered in name "Numerical Results" for its practical interest [7].
} 
Fig. 1 Architecture of the considered MG

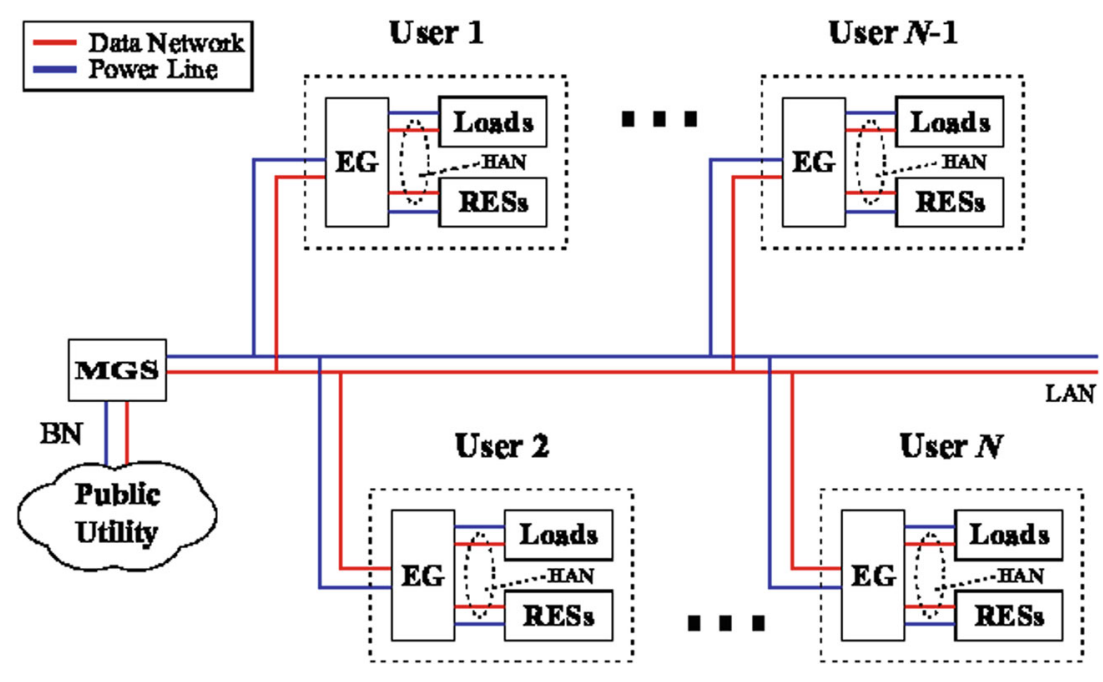

at any instant $t$; here $S_{m}(>0)$ represents the maximum power that can be absorbed from the public utility and

$S_{r} \triangleq \sum_{n=1}^{N} P_{g, \text { max }}^{(n)}<0$

is the maximum power (originating from the $\mathrm{MG}$ renewable energy sources) that can be delivered to the utility itself.

\section{Demand-Side Management Based on Bayesian Game Theory}

In this Section a brief description of our game model is provided and, on the basis of this model, a mixed strategy for the activation of SLs is developed.

\section{Rules and Description of the Game}

In the derivation of our strategy the following assumptions are made: 1) the EGs of the MG prosumers do not exchange information, but are able to listen to common signals, originating from the MG supervisor and providing proper statistical information about the expected future power consumption/generation in the MG;2) when an EG receives the request of activating a SL from the associated prosumer, it autonomously decides whether to turn the load itself on or not; 3 ) the time axis of any prosumer is divided in slots to ease the modellization of prosumer actions (the slot duration is denoted $T_{s}$ in the following).

Our main goal is mitigating PAR in load demand, while trying to satisfy most of (if not all) the requests of activation of SLs submitted by multiple MG prosumers to their EGs in a certain time interval. To reach this goal, we need to devise a management strategy for SLs such that: a) if multiple requests are submitted for turning them on in the same

$S_{r}<p_{T}(t)<S_{m}$ 
interval, their activations are properly scheduled; b) it operates in a distributed and noncooperative fashion, so that any explicit information sharing among the MG prosumers is avoided. To develop this strategy, in the following we focus on the management of the SLs owned by the $n$-th prosumer and assume that: a) this prosumer requests its EG the activation of its $l$-th SL (with $l \in\left\{0,1, \ldots, N_{s l}^{(n)}-1\right\}$, where $N_{s l}^{(n)}$ is the overall number of its SLs) at the time instant $t_{l, 0}^{(n)} ; \mathrm{b}$ ) if this SL was activated, this would entail the future absorption $^{3}$ of $P_{l}^{(n)} \mathrm{W}$ for $T_{l}^{(n)}$ s. The $n$-th prosumer's EG (the $n$-th EG, briefly), after receiving this request, has to face an activation dilemma, since it must select one of the following two options: a) satisfy this request; b) reject it (so that the SL activation is postponed). In our work this EG is modelled as a player (dubbed player \#1 in the following), behaving in a rational and selfish fashion, and whose action set consists of two distinct options, namely "turning the load on" (briefly, ON) or "keeping it off" (briefly, OFF). Moreover, since this EG competes with all the MG other prosumers in the exploitation of the available energy resources, the community of the remaining $(N-1)$ prosumers can be modelled as a single fictitious opponent, called player \#2 in the following and characterized by the overall power flow

$p_{-n}(t) \triangleq p_{T}(t)-p_{n}(t)=\sum_{\substack{l=1 \\ l \neq n}}^{N} p_{l}(t)$,

with

$P_{g, \text { max }}^{(-n)}<p_{-n}(t)<P_{a, \max }^{(-n)}$,

where $P_{a, \max }^{(-n)}>0$ and $P_{g, \max }^{(-n)} \leq 0$ represent the maximum absorbed and generated powers, respectively, of the opponent itself; note that $p_{-n}(t)>0(<0)$ if player \#2 absorbs power from (supplies power to) the MG. These considerations have led us to the conclusion that a two player game model, in place of a substantially more complicated $N$ player model, can be adopted to describe the interactions of the $n$-th prosumer with the rest of the MG. From the $n$-th EG (i.e., player \#1) perspective, a complete description of

\footnotetext{
${ }^{3}$ Note that a pessimistic model for the power consumption profile of the $l$-th SL is employed in our work for any $l$, since, in practice, $P_{l}^{(n)}$ represents the maximum power absorbed by such a SL in its activation interval. This model has been useful in our computer simulations, since it has allowed us to simplify the computation of the integrals involved in the strategy we propose. In principle, as it will become clearer in the following, more realistic profiles could be adopted in our strategy to improve its accuracy in the management of the MG SLs. However, this result would be achieved at the price of an increased computational cost, since the use of a smaller step size in all the numerical integrations in the time variable $t$ should be required.
}

this game requires the evaluation of the payoffs associated with the above mentioned couple of different actions. In the following, we assume that:

- If player \#1 decides to keep its $l$-th SL off, the payoff associated with this action is equal to 0 regardless of the power absorption/generation of the $n$-th prosumer and that of all the other prosumers. This choice is motivated by fact this action does not change the operating conditions of the MG.

- If player \#1 decides to turn its $l$-th SL on, the associated payoff $E P_{n}$ depends on the expected future behavior of both the $n$-th prosumer itself and its fictitious opponent (for this reason, $E P_{n}$ represents an expected payoff). In other words, in our model this payoff is influenced by the statistics of the future power consumption/generation characterizing the whole prosumer community.

The expression derived for $E P_{n}$ in the following part of this Section relies on:

1. A simple economic model (i.e., a pricing model) which establishes that the provision of a service, namely a power exchange between a prosumer and the MG, is paid or rewarded with an economic counterpart, represented by a certain amount of virtual currency, ${ }^{4}$ expressed in monetary units (mus).

2. The availability of some specific statistical information at the $n$-th EG.

\section{Economic Model}

As far as point 1) is concerned, we assume that any power exchange of player \#1 with the MG entails a variation in the total amount of virtual currency owned by itself. In our model such a variation depends by the MG state and a specific utility function (called cost function in the following). This functions describes the dependence of the rate of change of the overall amount of the virtual currency owned by player $\# 1$ on both the powers $p_{n}(t)$ and $p_{-n}(t)$. In particular, in the following it is assumed that:

- The proposed cost function is influenced by the MG operating conditions, which are represented by a single state variable; such a variable can take on two different values only, for simplicity. ${ }^{5}$ In particular, at any time

\footnotetext{
${ }^{4}$ Such a cost/profit can be certainly related to the real cost/profit but, generally speaking, is evaluated according to different rules, as explained in more detail below.

${ }^{5}$ Note that, as it will become clearer in the following, our approach can be easily extended to the case in which a larger number of MG states is assumed.
} 
instant $t$ the MG can operate in its normal state (briefly, state 0 ) characterized by

$p_{T}(t) \leq S_{c}$,

where $S_{c}$ denotes a proper positive power threshold, and a stress state (briefly, state 1) characterized by

$S_{c}<p_{T}(t)<S_{m}$.

In practice, the former state corresponds to normal operating conditions for the MG, whereas the latter one is characterized by a significant power absorption and, consequently, by some risk of blackout.

- The cost function is given by

$$
\begin{aligned}
C\left(p_{n} / p_{-n}\right) \triangleq & -\omega_{A}\left(p_{T}\right) \cdot \max \left(p_{n}, 0\right) \\
& -\omega_{G}\left(p_{T}\right) \cdot \min \left(p_{n}, 0\right) \\
& +\omega_{F}\left(p_{n}, p_{-n}\right) \cdot g\left(p_{n}, p_{-n}\right)
\end{aligned}
$$

and expresses, for a given $p_{-n}$ (i.e., for a given overall absorption/generation of player \#2) the cost (if negative) or reward (if positive) in mu/s associated with the power flow $p_{n} .{ }^{6}$ In the last expression the first term represents the cost associated with the power absorbed by player \#1 from the MG, the second one the profit coming from the power supplied by player \#1 to the MG, the third one is a fairness term relating the instantaneous power absorption/supply of player \#1 with that of player $\# 2$, and $\omega_{A}\left(p_{T}\right), \omega_{G}\left(p_{T}\right)$ and $\omega_{F}\left(p_{T}\right)$ are 3 distinct weight functions (expressed in $\mathrm{mu} / \mathrm{J}$ ), whose parameters can be adjusted by the MG supervisor (and broadcasted to all the prosumers) in order to influence prosumers' behavior and, in particular, to discourage them from buying and selling power at the same time in order to enrich themselves without giving a real power contribution to the MG.

In our model specific choices have been made for the functions appearing in Eq. 10. In particular, the expression

$\omega_{X}\left(p_{T}\right) \triangleq\left\{\begin{array}{c}\omega_{X}^{(i)} \text { for } p_{T} \leq S_{c} \\ \omega_{X}^{(i)}+\omega_{X}^{(i)}\left(p_{T}-S_{c}\right) / S_{c} \text { for } p_{T}>S_{c}\end{array}\right.$

with $X=A$ or $G$, where $i=0$ (1) in the normal (stress) state, and $\omega_{X}^{(0)}$ and $\omega_{X}^{(1)}$ denote positive parameters, has been selected for the weight functions of the first two terms contained in the right-hand side (RHS) of Eq. 10. This choice ensures that, for a given value of $p_{n}$, the cost or reward in $\mathrm{mu} / \mathrm{s}$ remains constant until the MG works in its normal state; on the contrary, if the MG enters its stress state (i.e., if $p_{T}$ crosses the threshold $S_{c}$ ), such a cost (reward) increases

\footnotetext{
${ }^{6}$ Note that in the following the dependence of powers on the time variable $t$ is often omitted to ease the reading.
}

linearly ${ }^{7}$ with the deviation of $p_{T}$ from $S_{c}$ in order to discourage (encourage) player \#1 to absorb further power from (supply further power to) the MG if $p_{n}>0\left(p_{n}<0\right)$. The selection of the third term of the RHS of Eq. 10 deserve various comments. First of all, in formulating a mathematical expression for this term, it has been assumed that it comes into play only if the overall power absorption or generation in the MG is significant and, in particular, if $\left|p_{T}\right|>S_{L}$, where $S_{L}$ is a positive power threshold not exceeding $S_{c}$; in other words

$g\left(p_{n}, p_{-n}\right)=0$

for $\left|p_{n}+p_{-n}\right| \leq S_{L}$. Moreover, the following two cases have been considered: a) $p_{n}$ and $p_{-n}$ have the same sign (i.e., $\left.\operatorname{sgn}\left(p_{n} p_{-n}\right)=1\right)$; b) $p_{n}$ and $p_{-n}$ have opposite signs. In case a), since both players are absorbing power from (or supplying power to) the MG, player \#1 should be discouraged to absorb (supply) further power in order to avoid an overload (energy overproduction). For this reason, in these conditions the fairness term should represent an additional cost charged to player \#1; in our model such a cost is assumed to be proportional to the product between the deviation of $\left|p_{T}\right|$ from $S_{L}$ (i.e., the difference $\left.\left(\left|p_{T}\right|-S_{L}\right)\right)$ and the ratio

$r\left(\tilde{p}_{n}, \tilde{p}_{-n}\right) \triangleq \frac{\left|\tilde{p}_{n}\right|}{\left|\tilde{p}_{n}\right|+\left|\tilde{p}_{-n}\right|}$,

which quantifies the fraction of power absorbed or supplied by player \#1 itself; here, $\tilde{p}_{n} \triangleq p_{n} / P_{a, \max }^{(n)}\left(\tilde{p}_{n} \triangleq\right.$ $\left.p_{n} / P_{g, \text { max }}^{(n)}\right)$ for $p_{n}>0\left(p_{n}<0\right)$ and $\tilde{p}_{-n} \triangleq p_{-n} / P_{a, \max }^{(-n)}$ $\left(\tilde{p}_{-n} \triangleq p_{-n} / P_{g, \max }^{(n)}\right)$ for $p_{-n}>0\left(p_{-n}<0\right)$ denote normalised powers. ${ }^{8}$ On the contrary, in case b) player \#1 should be encouraged to absorb (supply) further power from (to) the MG, since its opponent is supplying power to (absorbing power from) the MG and MG power needs should be satisfied as much as possible exploiting local renewable energy sources. For this reason, in these conditions the fairness term should represent a reward for player $\# 1$; in our model such a reward is assumed to be proportional to the product between the deviation $\left.\left(\left|p_{T}\right|-S_{L}\right)\right)$ and the function

$h\left(\tilde{p}_{n}, \tilde{p}_{-n}\right) \triangleq\left|\tilde{p}_{n} \cdot \tilde{p}_{-n}\right|$.

Note that the last factor ensures that, for a given $\tilde{p}_{n}$, the gain for player \#1 increases with $\left|\tilde{p}_{-n}\right|$; this means, for instance, that, if player \#1 is consuming a portion of the power generated by the renewable energy sources owned by player \#2,

\footnotetext{
${ }^{7}$ Other options can be considered for the function $\omega_{X}\left(p_{T}\right)(11)$; our simulation results have evidenced, however, that it achieves a good tradeoff between performance and computational complexity.

${ }^{8}$ Note that the use of such normalised powers is motivated by the need of putting the two players on the same footing in our game model when assessing fairness in the exploitation of energy resources.
} 
Fig. 2 Trend of the cost function $C\left(p_{n} / p_{-n}\right)(10)$ employed in our game model

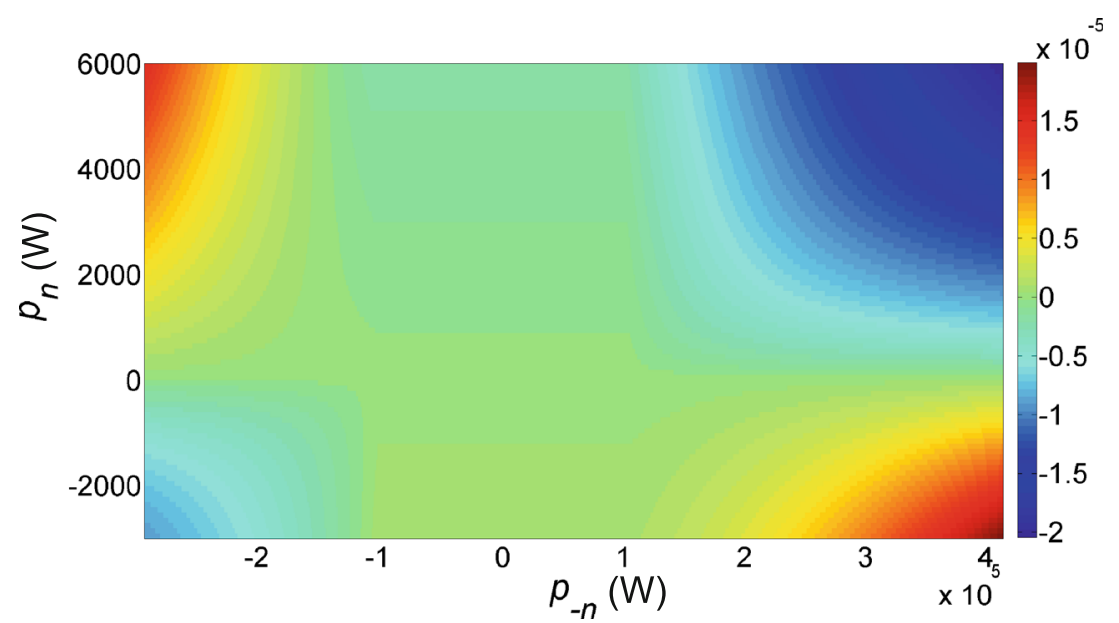

its reward will increases as this power gets larger. All these considerations lead to the expression

$$
\begin{aligned}
g\left(p_{n}, p_{-n}\right) \triangleq & -\operatorname{sgn}\left(p_{n} p_{-n}\right) u\left[\left|p_{n}+p_{-n}\right|-S_{L}\right] . \\
& {\left[\left|p_{n}+p_{-n}\right|-S_{L}\right] f\left(\tilde{p}_{n}, \tilde{p}_{-n}\right), }
\end{aligned}
$$

where $u[\cdot]$ represents the unit step function and

$f\left(\tilde{p}_{n}, \tilde{p}_{-n}\right) \triangleq\left\{\begin{array}{c}r\left(\tilde{p}_{n}, \tilde{p}_{-n}\right) \text { ifsgn }\left(p_{n} p_{-n}\right)=1 \\ h\left(\tilde{p}_{n}, \tilde{p}_{-n}\right) \text { elsewhere }\end{array}\right.$.

Moreover, to further stress the difference between the above mentioned cases a) and b), the factor $\omega_{F}\left(p_{n}, p_{-n}\right)$ appearing in Eq. 10 is defined as

$\omega_{F}\left(p_{n}, p_{-n}\right) \triangleq\left\{\begin{array}{c}\omega_{F, 0} \text { ifsgn }\left(p_{n} p_{-n}\right)=1 \\ \omega_{F, 1} \text { elsewhere }\end{array}\right.$,

where $\omega_{F, 0}$ and $\omega_{F, 1}$ are positive parameters. Figure 2 shows the typical trend exhibited by the cost function $C\left(p_{n} / p_{-n}\right)(10)$ when the choices (11), (13)-(16) are made (the values selected for the parameters $\omega_{A}^{(0)}, \omega_{A}^{(1)}, \omega_{G}^{(0)}, \omega_{G}^{(1)}$, $\omega_{F, 0}$ and $\omega_{F, 1}$ are listed in Table 1 of "Numerical Results"); in analysing this trend, it should be kept into account that the function $g\left(p_{n}, p_{-n}\right)$ takes on negative (positive) values, that is it is entails a reduction (increase) in the overall amount of stored virtual currency, when $p_{n}$ and $p_{-n}$ are concordant (discordant).

\section{Statistical Information Available to MG Prosumers}

As far as point 2) (i.e., the statistical information available to the $n$-th EG) is concerned, we assume that player \#1 is endowed with the knowledge of two different probability density functions (pdfs), one related to the overall power flow in the MG, the other one to the behavior exhibited by the $n$-th prosumer. In particular, the $n$-th EG statistical knowledge about the whole MG is condensed in:

1. The first order probability density function (pdf) $f_{p_{T}^{(i)}}(x ; \tau)$ (with $\tau>t$ ) referring to the overall power $p_{T}^{(i)}(t)$ absorbed by the MG or supplied to the public utility in the absence of DSM.

2. The first order pdf $f_{p_{n}^{(r)}}(x ; \tau)$ of the random portion $p_{n}^{(r)}(t)$ of $p_{n}(t)$ (see (2)).
Table 1 Values of the main parameters characterising the considered MG and the proposed DSM strategy

\begin{tabular}{llll}
\hline $\begin{array}{l}\text { MG } \\
\text { Parameter }\end{array}$ & Value & $\begin{array}{l}\text { Cost function } \\
\text { Parameter }\end{array}$ & Value \\
\hline$N$ & 100 & $\omega_{A}^{(0)}=\omega_{G}^{(0)}$ & $30 \mathrm{mu} / \mathrm{J}$ \\
$P_{g, \text { max }}^{(n)}$ & $-3 \mathrm{~kW}$ & $\omega_{A}^{(1)}=\omega_{G}^{(1)}$ & $200 \mathrm{mu} / \mathrm{J}$ \\
$P_{a, \text { max }}^{(n)}$ & $6 \mathrm{~kW}$ & $\omega_{F}^{(0)}$ & $10 \mathrm{mu} / \mathrm{J}$ \\
$S_{m}$ & $0.7 \cdot N \cdot P_{a, \text { max }}^{(n)} \mathrm{W}$ & $\omega_{F}^{(1)}$ & $4.5 \mathrm{mu} / \mathrm{J}$ \\
$S_{r}$ & $N \cdot P_{g, \text { max }}^{(n)}$ & $S_{c}$ & $S_{m}$ \\
$S_{L}$ & $0.25 \cdot S_{m} \mathrm{~W}$ & & \\
$T_{l}^{(n)}$ & $6 \mathrm{~h}$ & Activation probability & \\
$T_{D}$ & $24 \mathrm{~h}$ & Parameter & Value \\
$T_{S}$ & $15 \mathrm{mins}$ & $P_{S}$ & $0.90,0.95$ \\
$\delta$ & 0.75 & & \\
& & &
\end{tabular}


Actually, as it will become clearer below, what is really needed in our derivation of the payoff $E P_{n}$ is the knowledge of the joint pdf $f_{p_{n}^{(r)}, p_{-n}^{(i)}}(x, y ; \tau)$. In the following we assume that this pdf can be factored as

$f_{p_{n}^{(r)}, p_{-n}^{(i)}}(x, y ; \tau)=f_{p_{n}^{(r)}}(x ; \tau) f_{p_{-n}^{(i)}}(y ; \tau)$,

since the statistical behavior of $p_{-n}^{(i)}$ is influenced by that of a number of prosumers (forming player \#2), that, generally speaking, may exhibit different habits in terms of power consumption/generation than those characterising player \#1.

It is also important to point out that in any real world implementation of the proposed approach specific learning algorithms need to be developed for estimating the above mentioned pdfs on the basis of load/generation data. In particular, we note that:

- On the one hand, the pdf $f_{p_{T}^{(i)}}(x ; \tau)$ can be estimated by the MG supervisor and then its description (based on a parsimonious parametric representation of this function) can be periodically (e.g., once every hour or once every half an hour) broadcasted to all the prosumers. This task unavoidably requires the exact knowledge of the past consumption habits of all the prosumers and, in particular, of a) the time instants at which any prosumer would like to instantly turn on its SLs and b) the power absorption these activations would entail. For this reason, each of the MG EGs is expected to memorise the above mentioned data and periodically send them to the MG supervisor with a certain delay (so that the real time or day ahead loads of the MG prosumers remain unknown to potential eavesdroppers and privacy is substantially preserved). Once the required data have been acquired, refined machine learning tools and, in particular, regression models can be exploited by the MG supervisor to reliably predict the statistical behavior of a prosumer community on the basis of both these data and weather forecasts (e.g., [2, 8, 13, 18, 20, 21]).

- On the other hand, the estimation of the pdf $f_{p_{n}^{(r)}}(x ; \tau)$ can be accomplished by the $n$-th prosumer's EG storing its real time consumption data over a number of different days and exploiting machine learning tools for a reliable prediction of the statistics of $p_{n}^{(r)}(t)$.

- The approximation $f_{p_{-n}^{(i)}}(y ; \tau) \cong f_{p_{T}^{(i)}}(x ; \tau)$ can be adopted for the evaluation of the payoff $E P_{n}$ if the number $N$ of $\mathrm{MG}$ prosumers is large.

\section{Evaluation of the Expected Payoff}

Given $C\left(p_{n} / p_{-n}\right)(10)$ and the knowledge of the statistical information described above, the expected payoff $E P_{n}$ associated with the $\mathrm{ON}$ action can be evaluated as follows. First, the expected overall cost $E C_{n}\left(p_{n}^{(s)} ; t_{l, 0}^{(n)}, t_{l, 1}^{(n)}\right)$ charged to player \#1 in the interval $\left[t_{l, 0}^{(n)}, t_{l, 1}^{(n)}\right]$ ( with $t_{l, 1}^{(n)} \triangleq t_{l, 0}^{(n)}+T_{l}^{(n)}$ ) for its power flow is defined as the integral of the cost function $C\left(p_{n} / p_{-n}\right)(10)$, averaged with respect to both $p_{n}^{(r)}(t)$ and $p_{-n}^{(i)}(t)$, over the interval $\left[t_{l, 0}^{(n)}, t_{l, 1}^{(n)}\right]$, i.e. as (note that $p_{n}(t)<S_{m}-p_{-n}(t)$; see Eqs. 4 and 6)

$$
\begin{aligned}
& E C_{n}\left(p_{n}^{(s)} ; t_{l, 0}^{(n)}, t_{l, 1}^{(n)}\right) \\
& \triangleq \int_{\tau=t_{l, 0}^{(n)}}^{t_{l, 1}^{(n)}} \int_{x_{2}=P_{g, \max }^{(-n)}}^{S_{m}-P_{1}^{(n)}} \int_{x_{1}=P_{g, \text { max }}^{(n)}}^{\min \left(P_{a x}^{(n)}, S_{m}-x_{2}\right)} C\left(x_{1} / x_{2}\right) \\
& \cdot f_{p_{n}^{(r)}, p_{-n}^{(i)}}\left(x_{1}-p_{n}^{(s)}(\tau), x_{2} ; \tau\right) d x_{1} d x_{2} d \tau .
\end{aligned}
$$

Then, the expected payoff $E P_{n}$ associated with the ON action of player \#1 is defined asthe difference between the expected cost resulting from the activation of the considered load at $t=t_{l, 0}^{(n)}$ and that associated with keeping it off, i.e. as

$$
\begin{gathered}
E P_{n}\left(p_{n}^{(s)+}, p_{n}^{(s)-} ; t_{l, 0}^{(n)}, t_{l, 1}^{(n)}\right) \triangleq E C_{n}\left(p_{n}^{(s)+} ; t_{l, 0}^{(n)}, t_{l, 1}^{(n)}\right) \\
-E C_{n}\left(p_{n}^{(s)-} ; t_{l, 0}^{(n)}, t_{l, 1}^{(n)}\right),
\end{gathered}
$$

where $p_{n}^{(s)+}(t)$ and $p_{n}^{(s)-}(t)$ denote the function $p_{n}^{(s)}(t)$ (see Eq. 2) associated with the ON action and the OFF action, respectively. A simplified expression for $E P_{n}\left(p_{n}^{(s)+}, p_{n}^{(s)-} ; t_{l, 0}^{(n)}, t_{l, 1}^{(n)}\right)(20)$ can be derived as follows. First of all, substituting (18) in (19) yields

$$
\begin{gathered}
E C_{n}\left(p_{n}^{(s)} ; t_{l, 0}^{(n)}, t_{l, 1}^{(n)}\right) \\
\cong \int_{\tau=t_{l, 0}^{(n)}}^{t_{l, 1}^{(n)}} \int_{x_{2}=P_{a, \max }^{(-n)}}^{S_{m}-P_{p_{-n}^{(n)}}^{(n)}} f_{p_{2}}^{(i)}\left(x_{2} ; \tau\right) \\
\cdot \int_{x_{1}=P_{g, \max }^{(n)}\left(P_{a, \max }^{(n)}, S_{m}-x_{2}\right)}^{\min } C\left(x_{1} / x_{2}\right) \\
\cdot f_{p_{n}^{(r)}}\left(x_{1}-p_{n}^{(s)}(\tau) ; \tau\right) d x_{1} d x_{2} d \tau .
\end{gathered}
$$

A further simplification can be obtained by replacing $\min \left(P_{a, \max }^{(n)}, S_{m}-x_{2}\right)$ with $P_{a, \max }^{(n)}$ in the upper limit of the innermost integral appearing in the RHS of the last formula; note that this approximation is motivated by the fact that the integrand function takes on negligible values in the region which has been included in integration domain by modifying the above mentioned limit. This produces

$$
\begin{gathered}
E C_{n}\left(p_{n}^{(s)} ; t_{l, 0}^{(n)}, t_{l, 1}^{(n)}\right) \\
\cong \int_{\tau=t_{l, 0}^{(n)}}^{t_{l, 1}^{(n)}} \int_{x_{2}=P_{g, \text { max }}^{(-n)}}^{S_{m}-P_{p_{-n}^{(n)}}^{(n)}} f_{p_{2}^{(i)}}\left(x_{2} ; \tau\right) \\
\cdot \int_{x_{1}=P_{g, \text { max }}^{(n)}}^{P_{a, m a x}^{(n)}} C\left(x_{1} / x_{2}\right) \cdot f_{p_{n}^{(r)}}\left(x_{1}-p_{n}^{(s)}(\tau) ; \tau\right) d x_{1} d x_{2} d \tau .
\end{gathered}
$$

Finally, substituting (22) in the RHS of Eq. 20 yields, after some manipulation,

$$
\begin{gathered}
E P_{n}\left(p_{n}^{(s)+}, p_{n}^{(s)-} ; t_{l, 0}^{(n)}, t_{l, 1}^{(n)}\right) \\
\cong \int_{p_{-n}=P_{g, \text { max }}^{(-n)}}^{S_{m}-P_{a, n}^{(n)}} \beta\left(p_{-n}, p_{n}^{(s)+} p_{n}^{(s)-} ; t_{l, 0}^{(n)}, t_{l, 1}^{(n)}\right) d p_{-n},
\end{gathered}
$$


where

$$
\begin{gathered}
\beta\left(p_{-n}, p_{n}^{(s)+}, p_{n}^{(s)-} ; t_{l, 0}^{(n)}, t_{l, 1}^{(n)}\right) \\
\triangleq \int_{\tau=t_{l, 0}^{(n)}}^{t_{l, 1}^{(n)}} f_{p_{-n}^{(i)}}\left(p_{-n} ; \tau\right) \int_{x=P_{g, \max }^{(n)}}^{P_{a, \max }^{(n)}} C\left(x / p_{-n}\right) \\
{\left[f_{p_{n}^{(r)}}\left(x-p_{n}^{(s)+}(\tau) ; \tau\right)\right.} \\
\left.-f_{p_{n}^{(r)}}\left(x-p_{n}^{(s)-}(\tau) ; \tau\right)\right] d x d \tau
\end{gathered}
$$

can be interpreted as an expected cost density, since it shows how the expected overall cost $E P_{n}\left(p_{n}^{(s)+}, p_{n}^{(s)-} ; t_{l, 0}^{(n)}, t_{l, 1}^{(n)}\right)$ (23) distributes over the $p_{-n}$ axis in the reference interval $\left[t_{l, 0}^{(n)}, t_{l, 1}^{(n)}\right]$. Actually, in our work a generalized expression of $\beta\left(p_{-n}, p_{n}^{(s)+}, p_{n}^{(s)-} ; t_{l, 0}^{(n)}, t_{l, 1}^{(n)}\right)(24)$, including a discount factor $\delta(0<\delta<1)$ [5], has been employed. This choice is motivated by the fact that: a) in our model the game is repeated by player \#1 every $T_{S} \mathrm{~s}$ until the $l$-th SL is turned on or a maximum activation delay is reached; b) generally speaking, the activation interval of the considered load covers $N_{l}^{(n)}$ slots (i.e., $T_{l}^{(n)}=N_{l}^{(n)} T_{s}$ ); c) the quantity $\beta\left(p_{-n}, p_{n}^{(s)+}, p_{n}^{(s)-} ; t_{l, 0}^{(n)}, t_{l, 1}^{(n)}\right)(24)$ can be expressed as the sum of $N_{l}^{(n)}$ terms, each referring to a distinct time slot and to which a weight decreasing exponentially with the slot index can be assigned [5]. This leads to the new expression

$$
\begin{gathered}
\tilde{\beta}\left(p_{-n}, p_{n}^{(s)+}, p_{n}^{(s)-} ; t_{l, 0}^{(n)}, t_{l, 1}^{(n)}\right) \\
=\frac{1-\delta}{1-\delta_{l}^{(n)}} \sum_{z=0}^{N_{l}^{(n)}-1} \delta^{z} \\
\beta_{z}\left(p_{-n}, p_{n}^{(s)+}, p_{n}^{(s)-} ; t_{l, 0}^{(n)}\right) d p_{-n}
\end{gathered}
$$

for the expected cost density, where

$$
\begin{gathered}
\beta_{z}\left(p_{-n}, p_{n}^{(s)+}, p_{n}^{(s)-} ; t_{l, 0}^{(n)}\right) \\
\triangleq \beta\left(p_{-n}, p_{n}^{(s)+}, p_{n}^{(s)-} ; t_{l, 0}^{(n)}+z T_{s}, t_{l, 0}^{(n)}+(z+1) T_{s}\right) .
\end{gathered}
$$

In our game model the goal of player \#1 is the maximization of its expected payoff $E P_{n}$. For this reason, the optimal pure strategy for the $n$-th prosumer can be formulated as

$\hat{t}_{l, 0}^{(n)}=\underset{\tilde{t}_{l, 0}^{(n)} \in S_{0}^{(n)}}{\arg \max } E P_{n}\left(p_{n}^{(s)+}, p_{n}^{(s)-} ; \tilde{t}_{l, 0}^{(n)}, \tilde{t}_{l, 0}^{(n)}+T_{l}^{(n)}\right)$,

where $S_{0}^{(n)}=\left\{t_{p} \mid t_{p}=t_{l, 0}^{(n)}+p T_{s} ; p=0,1, \ldots\right\}$ is the set of all the possible activation instants. Note that the dependence of the expected payoff $E P_{n}\left(p_{n}^{(s)+}, p_{n}^{(s)-} ; t_{l, 0}^{(n)}, t_{l, 1}^{(n)}\right)$ on the power consumption originating from all the other prosumers makes the derivation of an equilibrium point for the strategy (27) a non trivial task.

In our work, however, we decided not to adopt the strategy expressed by Eq. 27 for the following reasons:

- As already mentioned above, an updated estimate of the pdf $f_{p_{-n}^{(i)}}(y ; \tau)$ is periodically broadcasted by the MG supervisor and, similarly, an estimate of the pdf $f_{p_{n}^{(r)}}(x ; \tau)$ is evaluated by the $n$-th EG at least on a daily basis. For this reason, the cost function appearing in the RHS of Eq. 27 may take on substantially different values if computed at different epochs and needs to be recomputed any time the above mentioned pdfs are updated.

- The $n$-th prosumer could be interested in the activation of multiple SLs it owns (e.g., an air conditioner and a PHEV) in the same time interval; this need should be efficiently managed.

These considerations and some previous work on node management in cooperative communications [17, Sec. 5] have led us to developing the mixed strategy illustrated in the next Paragraph.

\section{Proposed Mixed Strategy}

In our game model player \#1 repeats the game at the instants $t_{p}^{(n)}=t_{l, 0}^{(n)}+p T_{s}$, with $p=0,1, \ldots, K_{n}-1$, until it opts for the $\mathrm{ON}$ action or the maximum number $K_{n}$ of consecutive attempts is reached. In the $p$-th attempt (with $\left.p=0,1, \ldots, K_{n}-1\right)$ the selection of a specific action in the given action set is randomized and, in particular, is based on the probabilities $P_{o n}^{(n)}[p]$ and $\left(1-P_{o n}^{(n)}[p]\right)$ assigned to the ON and OFF actions, respectively; here $P_{o n}^{(n)}[p]$ represents the activation probability for the $n$-th prosumer in the considered attempt. It is important to point out that:

- Given the set of probabilities $\left\{P_{o n}^{(n)}[p] ; p=\right.$ $\left.0,1, \ldots, K_{n}-1\right\}$, the probability $P_{s}^{(n)}$ of a success (i.e., the probability that the $\mathrm{ON}$ action is selected in $K_{n}$ attempts) is given by

$$
P_{s}^{(n)}=P_{o n}^{(n)}[0]+\sum_{l=1}^{K_{n}-1} P_{o n}^{(n)}[l] \prod_{k=1}^{l-1}\left(1-P_{o n}^{(n)}[k]\right)
$$

- If $P_{o n}^{(n)}[p]$ did not change over the $K_{n}$ attempts, i.e. $P_{o n}^{(n)}[p]=P_{o n}^{(n)}$ for any $p$, the last formula would produce

$$
P_{s}^{(n)}=1-\left(1-P_{o n}^{(n)}\right)^{K_{n}},
$$

then, in this case, the activation probability

$$
P_{o n}^{(n)}=1-\left(1-P_{s}^{(n)}\right)^{1 / K_{n}} \cong \frac{P_{s}^{(n)}}{K_{n}}
$$

should be selected in each attempt to ensure a probability of success equal to $P_{S}^{(n)}$.

Our mixed strategy aims at adjusting the probabilities $\left\{P_{\text {on }}^{(n)}[p] ; p=0,1, \ldots, K_{n}-1\right\}$ in a way that the reduction in the expected payoff $E P_{n}\left(p_{n}^{(s)+}, p_{n}^{(s)-} ; t_{l, 0}^{(n)}, t_{l, 1}^{(n)}\right)$ (i.e., the decrease in the virtual currency owned by the $n$-th prosumer) evaluated on the basis of the expected cost density 
(25), is minimized, on the average, over the set of prosumers. The derivation of this strategy has been partially inspired by Sergi and Vitetta [17] and can be motivated as follows. To begin, let us define the daily average ( $t_{b}$ denotes the beginning of the considered day and $T_{D}=86400 \mathrm{~s}$ its duration)

$$
\begin{gathered}
\bar{\beta}\left(p_{-n}, p_{n}^{(s)+}, p_{n}^{(s)-}\right) \\
\triangleq \frac{1}{T_{D}} \int_{\tau=t_{b}}^{T_{D}+t_{b}} \tilde{\beta}\left(p_{-n}, p_{n}^{(s)+}, p_{n}^{(s)-} ; \tau, \tau+T_{l}^{(n)}\right) d \tau .
\end{gathered}
$$

of the expected cost density $\tilde{\beta}\left(p_{-n}, p_{n}^{(s)+}, p_{n}^{(s)-} ; t_{l, 0}^{(n)}, t_{l, 1}^{(n)}\right)$ (25) and the function

$$
\begin{gathered}
\varphi\left(p_{-n}, p_{n}^{(s)+}, p_{n}^{(s)-} ; t_{l, 0}^{(n)}, t_{l, 1}^{(n)}\right) \\
\triangleq \tilde{\beta}\left(p_{-n}, p_{n}^{(s)+}, p_{n}^{(s)-} ; t_{l, 0}^{(n)}, t_{l, 1}^{(n)}\right)-\bar{\beta}\left(p_{-n}, p_{n}^{(s)+}, p_{n}^{(s)-}\right),
\end{gathered}
$$

which expresses, for a given $p_{-n}$, the deviation of this expected cost density from its average. Then, the deviation of the expected payoff $E P_{n}\left(p_{n}^{(s)+}, p_{n}^{(s)-} ; t_{l, 0}^{(n)}, t_{l, 1}^{(n)}\right)$ from its daily average in the considered time interval $\left(t_{l, 0}^{(n)}, t_{l, 1}^{(n)}\right)$ can be expressed as

$$
\begin{gathered}
\Delta E P_{n}\left(p_{n}^{(s)+}, p_{n}^{(s)-} ; t_{l, 0}^{(n)}, t_{l, 1}^{(n)}\right) \\
\triangleq E P_{n}\left(p_{n}^{(s)+}, p_{n}^{(s)-} ; t_{l, 0}^{(n)}, t_{l, 1}^{(n)}\right)-\overline{E P} \\
\left.\triangleq P_{n}^{(s)+}, p_{n}^{(s)-}\right) \\
=\int_{p_{-n}=P_{g, \max }^{(n)}}^{S_{m}-P_{a, m a x}^{(n)}} \varphi\left(p_{-n}, p_{n}^{(s)+}, p_{n}^{(s)-} ; t_{l, 0}^{(n)}, t_{l, 1}^{(n)}\right) d p_{-n},
\end{gathered}
$$

where

$$
\begin{gathered}
\overline{E P}_{n}\left(p_{n}^{(s)+}, p_{n}^{(s)-}\right) \\
\triangleq \int_{p_{-n}=P_{g, \text { max }}^{(-n)}}^{S_{m}-P_{a, m}^{(n)}} \bar{\beta}\left(p_{-n}, p_{n}^{(s)+}, p_{n}^{(s)-}\right) d p_{-n} .
\end{gathered}
$$

The integration domain $\Lambda^{(n)}=\left[P_{g, \max }^{(-n)}, S_{m}-P_{a, \text { max }}^{(n)}\right]$ of the integral appearing in the RHS of Eq. 33 can be partitioned into the set

$$
\begin{gathered}
\Sigma^{(n)} \triangleq \\
\left\{p_{-n} \mid p_{-n} \in \Lambda^{(n)}, \varphi\left(p_{-n}, p_{n}^{(s)+}, p_{n}^{(s)-} ; t_{l, 0}^{(n)}, t_{l, 1}^{(n)}\right)<0\right\}
\end{gathered}
$$

and its complement $\overline{\Sigma^{(n)}}$ with respect to $\Lambda^{(n)}$; consequently, (33) can be rewritten as

$$
\begin{gathered}
\Delta E P_{n}\left(p_{n}^{(s)+}, p_{n}^{(s)-} ; t_{l, 0}^{(n)}, t_{l, 1}^{(n)}\right) \\
=\int_{\frac{\Sigma^{(n)}}{(n)}} \varphi\left(p_{-n}, p_{n}^{(s)+}, p_{n}^{(s)-} ; t_{l, 0}^{(n)}, t_{l, 1}^{(n)}\right) d p_{-n} \\
-\int_{\Sigma^{(n)}}\left|\varphi\left(p_{-n}, p_{n}^{(s)+}, p_{n}^{(s)-} ; t_{l, 0}^{(n)}, t_{l, 1}^{(n)}\right)\right| d p_{-n} .
\end{gathered}
$$

Note that the first term appearing in the RHS of the last expression, being positive, provides an improvement in terms of virtual currency, whereas the second one, namely

$$
-\int_{\Sigma^{(n)}}\left|\varphi\left(p_{-n}, p_{n}^{(s)+}, p_{n}^{(s)-} ; t_{l, 0}^{(n)}, t_{l, 1}^{(n)}\right)\right| d p_{-n},
$$

being of opposite sign, accounts for a loss of virtual currency. Let us now set an equilibrium point in our game model by defining a reference power level, denoted $\bar{P}_{r}$, for the overall power flow $p_{T}(t)$ (3) (and, consequently, for $p_{-n}(t)(6)$, since $\left.p_{-n}(t) \cong p_{T}(t)\right)$. Then, let us partition the integration domain $\Sigma^{(n)}(35)$ in the (not necessarily connected) sets

$\Sigma_{+}^{(n)} \triangleq\left\{p_{-n} \mid p_{-n} \in \Sigma^{(n)}, p_{-n}>\bar{P}_{r}\right\}$

and

$\Sigma_{-}^{(n)} \triangleq\left\{p_{-n} \mid p_{-n} \in \Sigma^{(n)}, p_{-n}<\bar{P}_{r}\right\}$

and define the error signal

$$
\begin{gathered}
e_{n}[p] \triangleq \int_{p_{-n} \epsilon \Sigma_{-}^{(n)}}\left|\varphi\left(p_{-n}, p_{n}^{(s)+}, p_{n}^{(s)-} ; t_{l, 0}^{(n)}, t_{l, 1}^{(n)}\right)\right| d p_{-n} \\
\quad-\int_{p_{-n} \in \Sigma_{+}^{(n)}}\left|\varphi\left(p_{-n}, p_{n}^{(s)+}, p_{n}^{(s)-} ; t_{l, 0}^{(n)}, t_{l, 1}^{(n)}\right)\right| d p_{-n} .
\end{gathered}
$$

It is important to point that:

- The two integrals appearing in the last formula represent the areas of specific regions subtended by the function $\varphi\left(p_{-n}, p_{n}^{(s)+}, p_{n}^{(s)-} ; t_{l, 0}^{(n)}, t_{l, 1}^{(n)}\right)(32)$, as exemplified by Fig. 3, which refers to the MG scenario described in "Numerical Results" and to a case in which a positive value is obtained for $e_{n}[p]$ (40).

- From the definition (40) it can be easily inferred that, if $e_{n}[p]$ is positive, the $n$-th prosumer expected loss associated with a MG power absorption larger than $\bar{P}_{r}$ (i.e., with a $\mathrm{MG}$ operating above its equilibrium point) is smaller than that expected in the opposite case.

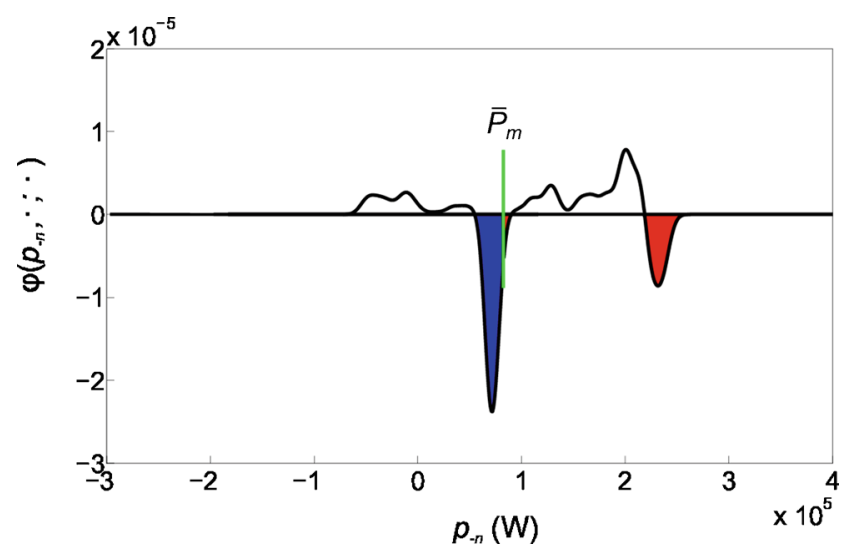

Fig. 3 Representation of the function $\varphi\left(p_{-n}, p_{n}^{(s)+}, p_{n}^{(s)-} ; t_{l, 0}^{(n)}, t_{l, 1}^{(n)}\right)$ (32) versus $p_{-n}$ for the recharge of a PHEV in a specific time interval (the scenario described in "Numerical Results" is considered). The reference power power threshold $\bar{P}_{r}$ and the areas measured by the two integrals appearing in the RHS of Eq. 40 are also shown. Note that in this case a positive value is obtained for $e_{n}[p]$, since the area associated with the domain $\Sigma_{+}^{(n)}$ (38) (area of the red region) is smaller than that associated with $\Sigma_{-}^{(n)}$ (39) (area of the blue region) 
Consequently, when $e_{n}[p]$ is positive, player \#1 should be encouraged to make the activation of its $l$-th SL more likely by increasing the activation probability $P_{o n}^{(n)}[p]$; of course, the opposite should occur when $e_{n}[p]$ is negative. These considerations suggest to develop a strategy based on the signal $e_{n}[p]$ for the adaptation of $P_{o n}^{(n)}[p]$ (with $\left.p=0,1, \ldots, K_{n}-1\right)$, which, on the basis of what has been just illustrated, should exhibit a monotonically increasing dependence on such a signal. In our work the formula

$P_{o n}^{(n)}[p]=\bar{P}_{n}+\gamma_{n} \tilde{e}_{n}[p]$

has been adopted for its simplicity; here, $\bar{P}_{n}$ defines a reference probability level, $\gamma_{n}$ is a real positive parameter and

$\tilde{e}_{n}[p] \triangleq \Phi_{n}\left(e_{n}[p]\right)$

where

$\Phi_{n}(e) \triangleq\left\{\begin{array}{c}-\bar{P}_{n} \gamma_{n}^{-1} \text { for } e<-\bar{P}_{n} \gamma_{n}^{-1} \\ e \text { for }-\bar{P}_{n} \gamma_{n}^{-1}<e<\left(1-\bar{P}_{n}\right) \gamma_{n}^{-1} \\ \left(1-\bar{P}_{n}\right) \gamma_{n}^{-1} \text { for } e>\left(1-\bar{P}_{n}\right) \gamma_{n}^{-1}\end{array}\right.$

represents a (prosumer-dependent) clipping function, introduced to limit the range of $P_{o n}[p]$, evaluated on the basis of Eq. 41 , to the interval $[0,1]$. The use of Eq. 41 requires the knowledge of the parameters $\bar{P}_{n}$ and $\gamma_{n}$. In practice, in our work

$\bar{P}_{n}=\frac{P_{s}^{(n)}}{K_{n}}$

has been selected for the reference probability level, as suggested by Eq. 30; in other words, the value assigned to $\bar{P}_{n}$ corresponds to that each element of the sequence $\left\{P_{o n}^{(n)}[p]\right\}$ should take on if all the attempts made by the $n$-th EG were equally likely (note that a large $K_{n}$ should be always expected). On the contrary, the optimization approach adopted in the evaluation of $\gamma_{n}$ is based on the following considerations. Substituting (42) in (41) and (41) in (28) yields the expression

$$
\begin{aligned}
P_{s}^{(n)} & =f_{s}\left(\bar{P}_{n}, \gamma_{n}\right)=\bar{P}_{n}+\gamma_{n} \Phi_{n}\left(e_{n}[0]\right) \\
& +\sum_{l}^{K_{n}-1}\left[\bar{P}_{n}+\gamma_{n} \Phi_{n}\left(e_{n}[l]\right)\right] \\
\cdot & \prod_{k=1}^{l-1}\left(1-\bar{P}_{n}-\gamma_{n} \Phi_{n}\left(e_{n}[k]\right)\right)
\end{aligned}
$$

which shows that the probability $P_{S}^{(n)}$ exhibits a complicated nonlinear dependence on the parameter $\gamma_{n}$ (note that a change in the value of this parameter modifies the thresholds appearing in the function $\left.\Phi_{n}(e)(43)\right)$. The relationship between the desired probability of success $P_{S}^{(n)}$ and the couple of parameters $\left(\bar{P}_{n}, \gamma_{n}\right)$ is exemplified by Fig. 4, which illustrates, for different values of $P_{s}^{(n)}$ and a specific sequence $\left\{e_{n}[l]\right\}$, the loci satisfying the condition $f_{s}\left(\bar{P}_{n}, \gamma_{n}\right)=P_{s}^{(n)}$. From this figure it can be easily inferred that, in the considered case, if $\bar{P}_{n}$ is selected according to Eq. 44, two distinct values of $\gamma_{n}$ (denoted $\gamma_{n}^{(0)}$ and $\gamma_{n}^{(1)}$ in the following, with $\left.\gamma_{n}^{(0)}<\gamma_{n}^{(1)}\right)$ satisfying the equality

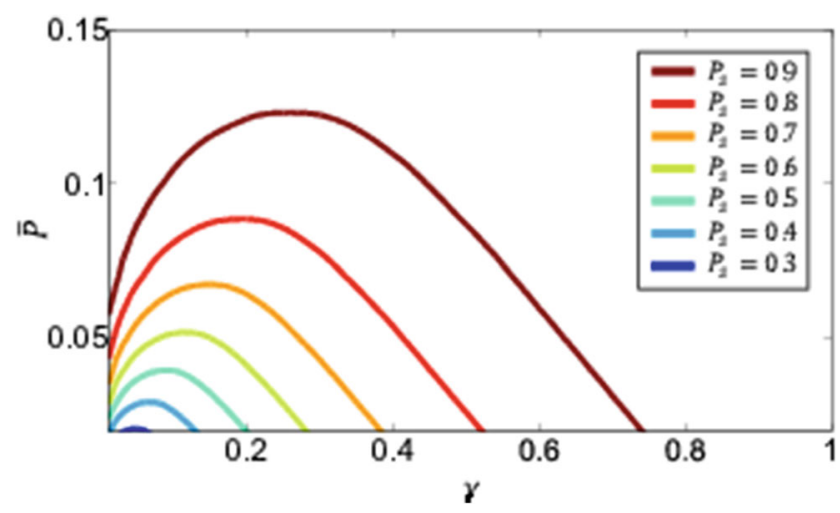

Fig. 4 Representation of the loci associated with a few values of $P_{s}^{(n)}$ in $\gamma_{n}-\bar{P}_{n}$ plane; a specific prosumer (i.e,. a specific value of $n$ ) is considered, but the prosumer index is omitted to ease the reading

$f_{s}\left(P_{s}^{(n)} K_{n}^{-1}, \gamma_{n}\right)=P_{s}^{(n)}$ are found. Note that these values lead to two substantially different sets of probabilities $\left\{P_{o n}^{(n)}[p] ; p=0,1, \ldots, K_{n}-1\right\}$. In our work $\gamma_{n}^{(1)}$ (i.e., the largest value of $\gamma_{n}$ ) has been always selected to emphasise the weight of the error $e_{n}[p]$ in the evaluation of $P_{o n}^{(n)}[p]$ on the basis of Eq. 41; in addition $\gamma_{n}^{(1)}$ has been evaluated by means of a simple direct search method [9]. Consequently, our strategy consists of the following steps:

1. Assign the desired value to the success probability $P_{S}^{(n)}$.

2. Evaluate $\bar{P}_{n}=P_{s}^{(n)} / K_{n}$ (see Eq. 44).

3. Compute the elements of the set $\left\{e_{n}[p], p=0,1, \ldots, K_{n}-1\right\}$ by means of Eqs. 24-26, (31), (32), (38)-(40).

4. Evaluate $\gamma_{n}$ by solving the equation $P_{s}^{(n)}=$ $f_{s}\left(\bar{P}_{n}, \gamma_{n}\right)$ (see Eq. 45).

5. Compute the elements of the set $\left\{\tilde{e}_{n}[p]=\Phi_{n}\left(e_{n}[p]\right), p=0,1, \ldots, K_{n}-1\right\} \quad$ (the value of $\gamma_{n}$ evaluated in the previous step is used in Eq. 43).

6. Set the time index $q$ to zero.

7. Compute the activation probability $P_{o n}^{(n)}[q]$ using Eq. 41.

8. Generate a random number $r_{n}$ belonging to the interval $[0,1]$.

9. If $r_{n}<P_{o n}^{(n)}[q]$, turn the $l$-th SL on (so that the activation procedure is over); otherwise, keep this SL off and proceed with the next step.

10. Increase the index $q$ by one and compare it with $K_{n}$. If $q$ is less than $K_{n}$, wait for $T_{s}$ s and go back to step 7) in order to repeat the game; otherwise (i.e., if $q=K_{n}$ ) assign new values to the parameters $P_{s}^{(n)}$ and $K_{n}$ and restart the game from step 1), if desired.

When using this strategy, the following considerations should be always kept into careful consideration: 
1. In any MG a certain number of types of SLs can be envisaged, where a type could be uniquely identified by the couple (absorbed power, absorption duration). If the $n$-th prosumer wants to turn on $N_{t}^{(n)}$ distinct SLs (belonging, generally speaking to different types) in the same time interval, its EG is expected to evaluate $N_{t}^{(n)}$ distinct probabilities (41), one for each of them. Note that turning a specific SL on entails an abrupt change in the function $p_{n}^{(s)}(t)$; consequently, the probabilities for all the other SLs waiting for activation need to be recomputed when this occurs.

2. The game is repeated once every $T_{s}$ sec. In principle, the value selected for the slot interval $T_{S}$ should be proportional to the correlation time of the overall power $p_{T}(t)$ of the MG; this indication is motivated by the fact that, if the OFF action has been selected by a given prosumer in a certain repetition of its game, the next attempt should be made when the overall power flow in MG has undergone a significant change.

3. The energy resources available in a MG are shared on the basis of a probabilistic mechanism; in principle, this does not ensure that the important constraint $p_{T}(t)<S_{m}$ (see Eq. 4) is met at an arbitrary instant. When SL scheduling is not sufficient to avoid the risk of an overload in the MG, the MG supervisor is expected to broadcast a message of selective detachment to all the MG prosumers, in order to require the immediate disconnection of a given portion of (if not all) the active SLs from the power grid.

4. The reference power level $\bar{P}_{r}$ plays an important role in game evolution, since a change in its value (which is selected by the MG supervisor and broadcasted to all the prosumers) modifies the equilibrium of the whole MG. It is reasonable to fix the value of this threshold on the basis of the consumption expected over the whole day; in practice, exploiting the available statistical information, the MG supervisor can evaluate the overall energy consumption expected in the considered day and, consequently, can obtain the average overall power expected in the same day. Following these indications, in our simulation $\bar{P}_{r}$ has been updated on a daily basis and in the $k$-th day

$$
\begin{gathered}
\bar{P}_{r}=P_{a v}[k] \\
\triangleq \frac{1}{T_{D}} \int_{\tau=t_{b}[k]}^{T_{D}+t_{b}[k]} \int_{x=P_{g, \max }}^{P_{a, \max }} x f_{p_{T}^{(i)}}(x ; \tau) d x d \tau
\end{gathered}
$$

has been selected; here, $P_{a v}[k]$ represents the average power flow in the whole MG expected over the considered day and $t_{b}[k]$ denotes the beginning of the day itself. This choice is motivated by the fact that: a) the average daily requests of the MG prosumers should be really satisfied through proper scheduling of their
SLs; b) limited fluctuations of the overall power $p_{T}(t)$ should be expected in the MG operating conditions associated with this equilibrium point (in other words, the MG PAR should be mitigated).

Finally, it is important to point out that providing an accurate estimate of the computational complexity of the proposed strategy is not easy. In fact, the most computationally efficient task in the proposed procedure is represented by the computation of the error signal $e_{n}[p]$ (with $p=0,1, \ldots, K_{n}-1$ ) on the basis of Eq. 40. This requires the integration of the function $\varphi\left(p_{-n}, p_{n}^{(s)+}, p_{n}^{(s)-} ; t_{l, 0}^{(n)}, t_{l, 1}^{(n)}\right)(32)$ over the time and user dependent domains $\Sigma_{+}^{(n)}$ (38) and $\Sigma_{-}^{(n)}$ (39); in turn, this function depends on $\tilde{\beta}\left(p_{-n}, p_{n}^{(s)+}, p_{n}^{(s)-} ; t_{l, 0}^{(n)}, t_{l, 1}^{(n)}\right)$ (25) and $\bar{\beta}\left(p_{-n}, p_{n}^{(s)+}, p_{n}^{(s)-}\right)$ (31), which involve two dimensional and three dimensional integrations, respectively, of the same function. This leads to the conclusion that the complexity of our strategy is dominated by the four dimensional integral resulting from the integration of $\bar{\beta}\left(p_{-n}, p_{n}^{(s)+}, p_{n}^{(s)-}\right)$ in the computation of Eq. 40. For this reason, in order to estimate such a complexity, an upper bound on the number of floating point operations (FLOPs) required for this integration ${ }^{9}$ has been assessed in the specific scenario considered in "Numerical Results". In doing so, distinct weights have been assigned to different types of operations; in particular, the weights $\{3,3,2,2,1,0\}$ have been assigned to exponentials, roots, divisions, multiplications, additions and comparisons, respectively.

\section{Numerical Results}

The performance of our strategy has been assessed by means of computer simulations for a MG collecting $N=100$ residential prosumers. In all our simulations the following assumptions have been made:

1. Each prosumer has subscribed a contract for the supply of $P_{a, \max }^{(n)}=6 \mathrm{~kW}$, of which $3.6 \mathrm{kWh}$ are explicitly devoted to the recharge of its PHEV (which is assumed to be its only SL for simplicity and requires $6 \mathrm{~h}$ for a full recharge). Moreover, it is able to generate up to $-P_{g, \text { max }}^{(n)}=3 \mathrm{~kW}$ thanks to its photovoltaic panels. The power level generated by the photovoltaic panels of a given prosumer in a specific day has been obtained by superimposing the average power profile for these renewable energy sources in the considered season (spring in our simulations) with a zero mean random

\footnotetext{
${ }^{9}$ This bound refers to the case in which both the integration domains $\Sigma_{+}^{(n)}(38)$ and $\Sigma_{-}^{(n)}(39)$ include all possible values of $p_{-n}$.
} 
Gaussian process (in practice, an auto-regressive model of order one has been adopted) to account for daily fluctuations.

2. Each prosumer owns 15 home appliances, each characterized by a specific probability mass function (pmf). The pmf associated with a specific appliance describes its activation probability over a single day (a $15 \mathrm{~m}$ step size has been used along the time axis), is prosumer independent and is based on typical behaviors of home users. In practice, the set of all the pmfs defines the prosumer profile of daily power consumption; a specific realization of the daily power consumption for a $\mathrm{MG}$ prosumer is shown in Fig. 5 (a $1 \mathrm{~h}$ step size is used in this representation to ease the reading). Note, however, that a Gaussian model has been adopted for approximating $f_{p_{n}^{(r)}}(x ; \tau)$, since it provides an accurate and parsimonious representation of this pdf; its parameters (mean value and variance) are perfectly known to the $n$ th prosumer for any $n$, so that an ideal knowledge of the pdf $f_{p_{n}^{(r)}}(x ; \tau)$ is available to its EG.

3. A Gaussian model has been also adopted for the pdf $f_{p_{T}^{(i)}}(x ; \tau)$ referring to the overall power flow of the MG in the absence of DSM; its parameters (mean value and variance) are perfectly known to all the prosumers, that employ the approximation $f_{p_{-n}^{(i)}}(y ; \tau) \cong$ $f_{p_{T}^{(i)}}(x ; \tau)$, as already mentioned in the previous Section.

4. The values of the parameters listed in Table 1 have been adopted by all the prosumers for the proposed DSM strategy.

5. In each simulation run, MG load demand has been observed over 3 consecutive days (i.e., $72 \mathrm{~h}$ corresponding to 288 slot intervals, each of $15 \mathrm{~min}$ ), so that a sort of steady state condition has been achieved in the middle (second) day; the recharge requests have been concentrated in the second day for all the PHEVs in order to assess the efficacy of the proposed DSM strategy in the presence of a relevant load demand. Moreover, in each simulation run, new realizations for the random components $\left\{p_{n}^{(r)}(t), n=0,1, \ldots, N-1\right\}$ of prosumer power and for the instants $\left\{t_{0}^{(n)}, n=0,1, \ldots, N-1\right\}$ at which the PHEV recharge requests are submitted, have been generated.

The selection of the probability $P_{s}^{(n)}$ and the evaluation of the related parameter $K_{n}$ for the $n$-th customer (see Paragraph 3.5) deserve the following comments. On the one hand, the value of $P_{s}^{(n)}$ should be chosen on the basis of a fairness criterion; more specifically, customers accepting a longer waiting time for the recharge of their PHEV should be satisfied with higher probability. On the other hand, the value of the parameter $K_{n}$ is influenced not only by such a waiting time, but also by the selection of the instant $t_{\max }^{(n)}$ within which the recharge of the PHEV for the $n$-th prosumer (with $n=0,1, \ldots, N-1$ ) should be completed; in fact, the parameter $K_{n}$ represents the number of time slots contained in the interval starting at $t=t_{p}^{(n)}$ (instant at which the $p$-th repetition of the game occurs) and ending at $t=t_{\max }^{(n)}$. In our simulations the values of $P_{s}^{(n)}$ and $t_{\max }^{(n)}$ have been selected on the basis of the interval $\left[t_{i}, t_{f}\right]$ in which the $n$-th prosumer requires the recharge of its PHEV to start; in particular, the following 4 distinct possibilities have been envisaged for all the prosumers: 1) $P_{s}^{(n)}=0.9$ and $t_{\max }^{(n)}=17: 00$ if $\left.\left[t_{i}=0: 00, t_{f}=7: 00\right] ; 2\right)$ $P_{s}^{(n)}=0.9$ and $t_{\max }^{(n)}=24: 00$ if $\left[t_{i}=7: 00, t_{f}=14: 00\right]$; 3) $P_{s}^{(n)}=0.95$ and $t_{\max }^{(n)}=7: 00$ of the next day if $\left[t_{i}=\right.$ $\left.14: 00, t_{f}=20: 00\right]$; 4) $P_{s}^{(n)}=0.95$ e $t_{\max }^{(n)}=11: 00$ of the next day if $\left[t_{i}=20: 00, t_{f}=24: 00\right]$. Moreover, in our simulations, if the recharge of a given PHEV had not

Fig. 5 Representation of a specific realization for the daily power consumption associated with 15 home appliances (unshiftable loads) owned by a MG prosumer
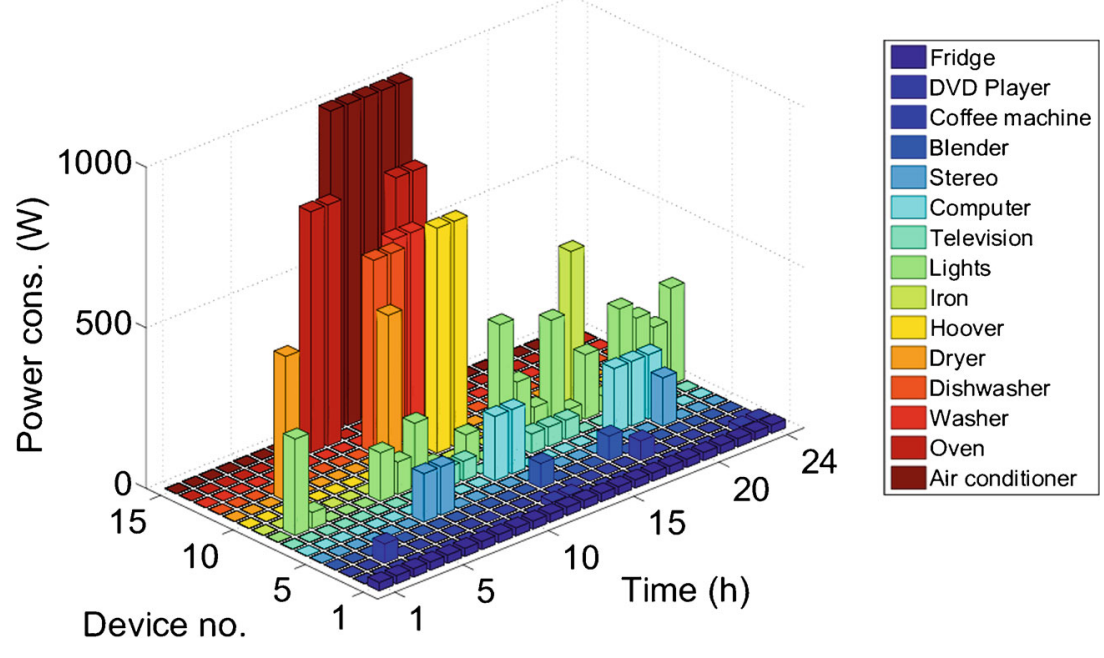
started (finished) yet at $t=t_{\max }^{(n)}$, the game has been repeated (the recharge has been continued) until that PHEV has been fully recharged; in doing so the values of $P_{s}^{(n)}$ and $K_{n}$ have remained unchanged.

Some numerical results are illustrated in Figs. 6, 7, 8 and 9. In particular, in Fig. 6 a sample function of the overall power $p_{T}(t)$ (blue curve) absorbed (if positive) by the MG prosumers from the public utility or delivered (if negative) by the MG to the utility itself is illustrated for the considered three day interval in the presence (lower picture) and in the absence (upper picture) of the developed DSM strategy; in both cases the overall power $p_{P H E V}(t)$ (red curve) absorbed by the PHEVs only is also shown. These results evidence that the scheduling of PHEVs may substantially lower the peaks in load demand due to SLs. This conclusion is also supported by Fig. 7 that shows the pdf $f_{P A R}(x)$ of the percentage improvement in the MG PAR due to DSM with respect to the case in which DSM is not employed. In fact, these results show that the use of DSM yields a $34 \%$ average improvement in the MG PAR; note that this improvement is substantially better than that provided by the game theoretic distribute strategy developed in Mohsenian-Rad et al. [11], where a $17 \%$ improvement is achieved in a different scenario.

Figure 8 shows the pdfs $f_{D}(x)$ of the waiting time $D$ (expressed in number of time slots) in the activation of PHEVs. In particular, the 4 upper figures refer to the 4 distinct intervals $\left[t_{i}, t_{f}\right]$ in which the prosumers require the recharge of their PHEVs to start (i.e., $\left[t_{i}=0: 00, t_{f}=\right.$ 7:00], $\left[t_{i}=7: 00, t_{f}=14: 00\right],\left[t_{i}=14: 00, t_{f}=\right.$ 20:00] and $\left.\left[t_{i}=20: 00, t_{f}=24: 00\right]\right)$; the lower figure, instead, refers to the whole day. It is important to note that:
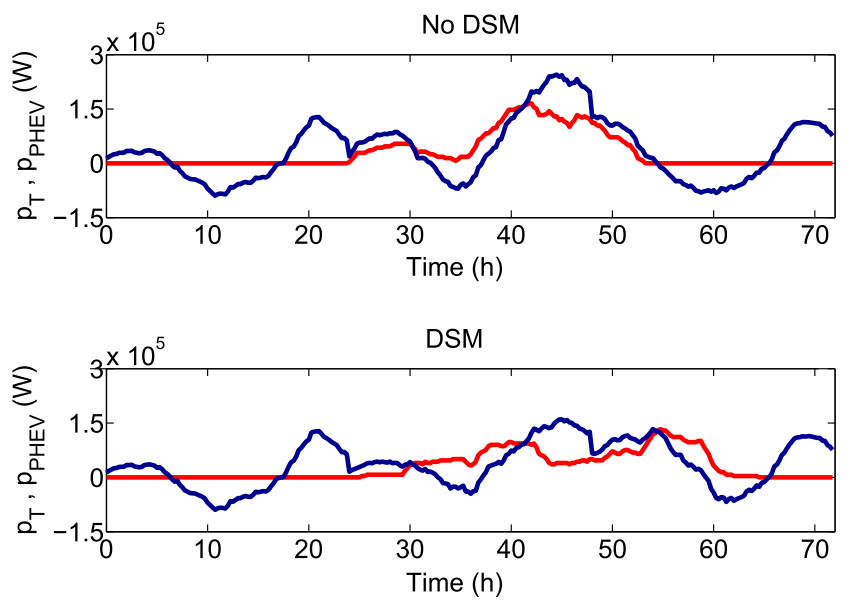

Fig. 6 MG overall power $p_{T}(t)$ (blue curve) and overall power $p_{P H E V}(t)$ (red curve) absorbed by the PHEVs over a three day inter$\mathrm{val}$ in the absence (upper picture) and in the presence (lower figure) of DSM

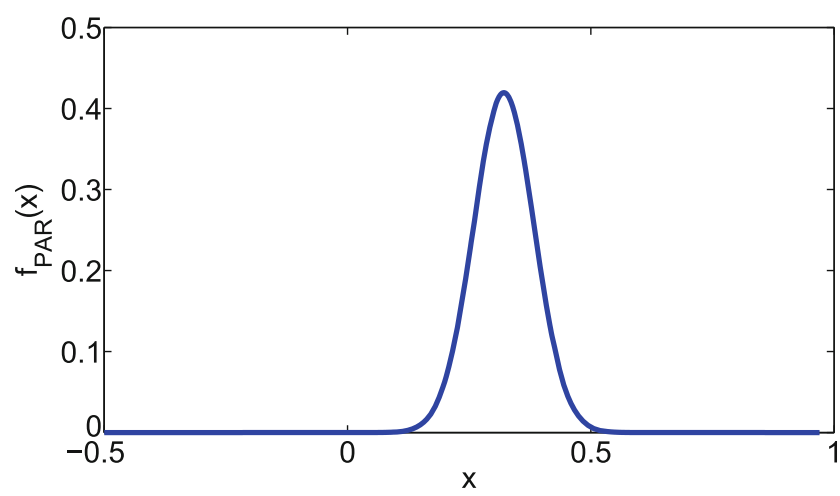

Fig. 7 Pdf $f_{P A R}(x)$ of the percentage improvement in the MG PAR due to the proposed DSM strategy

- The largest average delay is experienced by a PHEV in the intervals [14:00, 20:00] and [20:00, 24:00] in which most of load demand is concentrated.

- The delay distribution is influenced not only by the overall load demand, but also by the power limit $P_{a, \max }^{(n)}$ set on the power consumption of each prosumer; in fact, a SL cannot be turned on by the $n$-th prosumer if its power limit $P_{a, \max }^{(n)}$ is exceeded because of this new activation.

Further simulation results have evidenced that:

1. In the considered scenario the percentage of prosumers for whom the PHEV recharge has been completed within $t_{\max }^{(n)}$ are: a) $91.3 \%$ for the requests submitted in the intervals $\left[t_{i}=0: 00, t_{f}=7: 00\right]$; b) $96.7 \%$ in the interval $\left[t_{i}=20: 00, t_{f}=24: 00\right]$; c) $91.2 \%$ in the
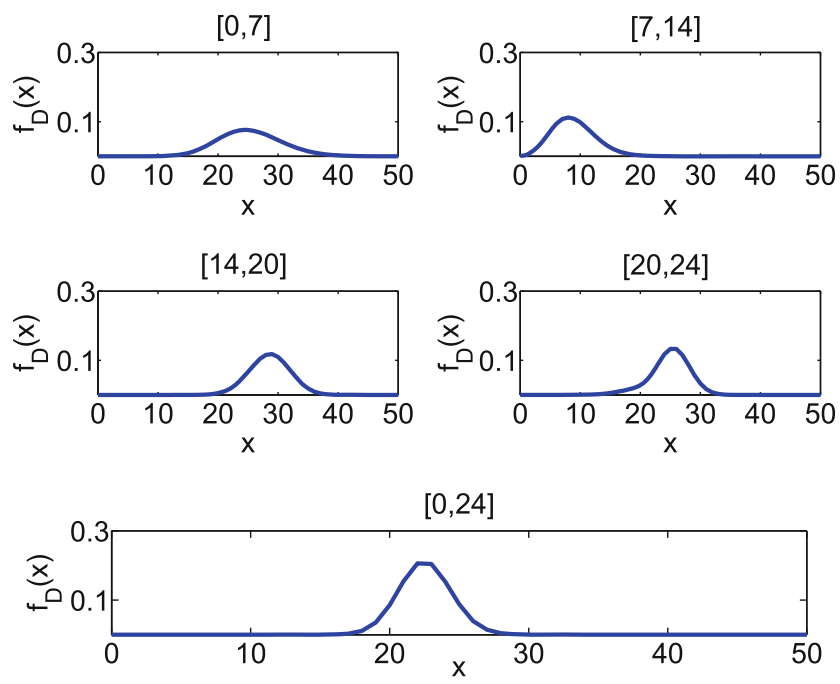

Fig. 8 Pdf $f_{D}(x)$ of the waiting time $D$ (expressed in number of time slots) in the activation of the PHEVs. The 4 upper figures refer to the $\left[t_{i}=0: 00, t_{f}=7: 00\right],\left[t_{i}=7: 00, t_{f}=14: 00\right],\left[t_{i}=14: 00, t_{f}=\right.$ 20:00] and $\left[t_{i}=20: 00, t_{f}=24: 00\right]$; the lower figure, instead, refers to the whole day 
Fig. 9 Realization of the expected payoffs

$\left\{E P_{n}, n=1,2, \ldots, 100\right\}$

evaluated for the activation of the PHEVs owned by all the MG prosumers in the presence (blue dots) and in the absence (red dots) of DSM

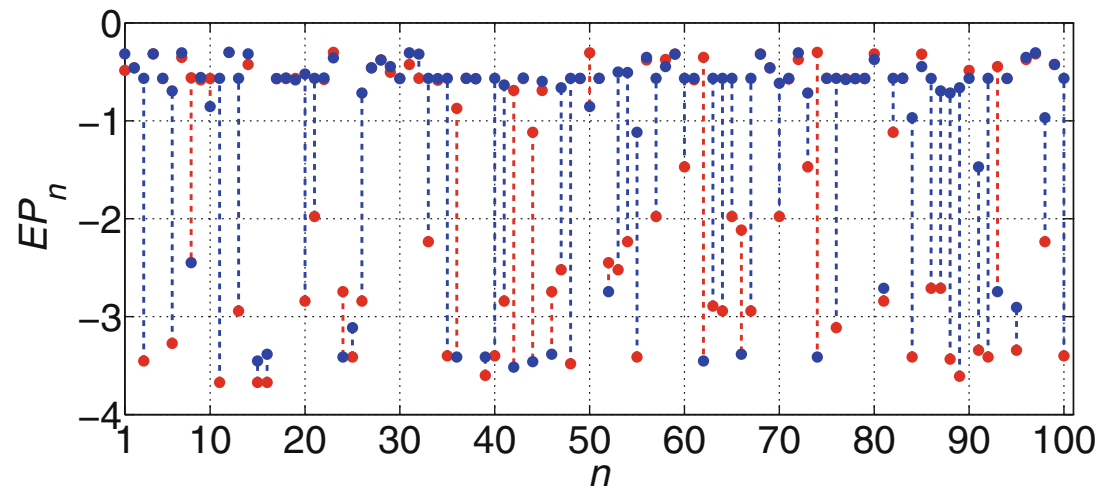

interval $\left[t_{i}=7: 00, t_{f}=14: 00\right]$; d) $97.0 \%$ in the interval $\left[t_{i}=14: 00, t_{f}=20: 00\right]$. These percentages have been estimated by averaging the data acquired over 100 distinct days of the same season.

2. The PAR improvement is influenced by the discount factor $\delta$; in the considered scenario optimal performance is obtained for $0.6<\delta<0.8$ (for this reason, $\delta=0.75$ has been selected in generating the data shown in this Section).

3. The gap between the expected payoff $E P_{n}$ (20) evaluated for the activation of the PHEV owned by the $n$-th prosumer in the presence of the proposed DSM strategy and that computed in the absence of DSM may exhibit significant variations from prosumer to prosumer. This is exemplified by Fig. 9, which shows a realization of the values of the above mentioned expected payoffs $\left\{E P_{n}, n=1,2, \ldots, 100\right\}$ evaluated in the presence of DSM (blue dots) and in the absence of it (red dots) for all the MG prosumers; note that the line that connects the two dots referring to a specific user is blue (red) if the first value is greater (smaller) than the second one. These results show that, in this specific case, 78 prosumers of the whole community benefit from the DSM strategy in terms of virtual currency. Extensive simulations have also evidenced that, on the average, $75 \%$ of the prosumer population gets an improvement in the expected payoff thanks to the use of the DSM strategy; in particular, the mean expected payoff for the PHEV activation is $-0.84 \mathrm{mu}$ in the presence of DSM and -1.31 $\mathrm{mu}$ in the absence of it.

Finally, as already mentioned in the previous Section, an upper bound on the computational complexity has been evaluated in the considered scenario under the assumptions that the following step sizes are used in all the numerical integrations involved in the evaluation of $e_{n}[p](40)$ : $\Delta_{t}=15 \mathrm{~m}, \Delta_{p_{-n}}=1 \mathrm{~kW}$ and $\Delta_{p_{n}}=100 \mathrm{~W}$ for the time $(t), p_{n}$ and $p_{-n}$ variables, respectively. This has led us to the conclusion that, in the considered scenario, the evaluation of the set of errors $\left\{e_{n}[p] ; p=0,1, \ldots, K_{n}-1\right\}$ requires less than 15 GFlops; we believe that, since this computation has to be repeated every $15 \mathrm{~m}$ in our case, the assessed complexity could be managed by low cost programmable hardware platforms available on the market.

\section{Conclusions}

In this manuscript a distributed DSM strategy relying on statistical information about prosumer consumption and the overall consumption of a MG has been developed. The proposed strategy allows to schedule SLs in way to mitigate fluctuations in load demand, while substantially preserving user privacy. Numerical results referring to the use of our strategy in the management of the recharge of PHEVs evidence that a significant reduction in the MG PAR can be achieved. Ongoing research work concerns the investigation of the effects of a mismatch between the real statistics of load demand/power generation and those available to the prosumers for the evaluation of their expected payoffs, and the management of energy storage units in a MG.

Acknowledgments This work has been accomplished in the framework of a PhD program organized by the Global Grant Spinner 2013, and funded by the European Social Fund and the Emilia Romagna Region.

\section{References}

1. Atzeni I, Ordonez L, Scutari G, Palomar D, Fonollosa J (2013) Noncooperative and cooperative optimization of distributed energy generation and storage in the demand-side of the smart grid. IEEE Trans Signal Proces 61(10):2454-2472

2. Couceiro M, Ferrando R, Manzano D, Lafuente L (2012) Stream analytics for utilities. Predicting power supply and demand in a smart grid. In: 2012 3rd international workshop on cognitive information processing (CIP), pp 1-6

3. Farhangi $H$ (2010) The path of the smart grid. IEEE Power and Energy Magazine 8(1): 18-28

4. Fouda M, Fadlullah Z, Kato N, Takeuchi A, Nozaki Y (2012) A novel demand control policy for improving quality of power usage 
in smart grid. In: 2012 IEEE Global Communications Conference (GLOBECOM), pp 5154-5159

5. Fudenberg D (1998) The theory of learning in games. MIT Press, Cambridge

6. Giannakis G, Kekatos V, Gatsis N, Kim S-J, Zhu H, Wollenberg B (2013) Monitoring and optimization for power grids: a signal processing perspective. IEEE Signal Proc Mag 30(5):107128

7. Green R, Wang L, Alam M (2010) The impact of plug-in hybrid electric vehicles on distribution networks: a review and outlook. In: 2010 IEEE power and energy society general meeting, pp 1-8

8. Hong T, Wilson J, Xie J (2014) Long term probabilistic load forecasting and normalization with hourly information. IEEE Transactions on Smart Grid 5(1):456-462

9. Kolda TG, Lewis RM, Torczon V (2003) Optimization by direct search: new perspectives on some classical and modern methods. SIAM Rev 45(3):385-482

10. Li D, Jayaweera S (2015) Distributed smart-home decisionmaking in a hierarchical interactive smart grid architecture. IEEE Trans Parallel Distrib Syst 26(1):75-84

11. Mohsenian-Rad A-H, Wong V, Jatskevich J, Schober R, LeonGarcia A (2010) Autonomous demand-side management based on game-theoretic energy consumption scheduling for the future smart grid. IEEE Trans Smart Grid 1(3):320-331

12. Pedrasa M, Spooner T, MacGill I (2009) Scheduling of demand side resources using binary particle swarm optimization. IEEE Trans Power Systems 24(3):1173-1181

13. Qingfeng T, Jianhua Z, Zhengyong X (2014) Short-term microgrid load forecast method based on emd-kelm-ekf. In: 2014 International conference on intelligent green building and smart grid (IGBSG), pp 1-4

14. Ramachandran B, Srivastava S, Cartes D (2012) Decentralized congestion management in stochastic electric power markets with phev penetration. In: 2012 IEEE power and energy society general meeting, pp 1-6

15. Saad W, Han Z, Poor H, Basar T (2011) A noncooperative game for double auction-based energy trading between phevs and distribution grids. In: 2011 IEEE international conference on smart grid communications (SmartGridComm), pp 267-272

16. Saad W, Han Z, Poor H, Basar T (2012) Game-theoretic methods for the smart grid: an overview of microgrid systems, demand-side management, and smart grid communications. IEEE Signal Proc Mag 29(5):86-105

17. Sergi S, Vitetta G (2010) A game theoretical approach to distributed relay selection in randomized cooperation. IEEE Trans Wirel Commun 9(8):2611-2621

18. Shafiullah G, Oo A, Jarvis D, Ali A, Wolfs P (2010) Potential challenges: Integrating renewable energy with the smart grid. In: 2010 20th Australasian Universities power engineering conference (AUPEC), pp 1-6

19. Shao S, Pipattanasomporn M, Rahman S (2009) Challenges of phev penetration to the residential distribution network. In: PES '09 Power energy society general meeting, 2009. IEEE, pp 1-8

20. Sharma N, Sharma P, Irwin D, Shenoy P (2011) Predicting solar generation from weather forecasts using machine learning. In: 2011 IEEE international conference on smart grid communications (SmartGridComm), pp 528-533

21. Tastu J, Pinson P, Trombe P-J, Madsen H (2014) Probabilistic forecasts of wind power generation accounting for geographically dispersed information. IEEE Transactions on Smart Grid 5(1):480-489

22. Vojdani A (2008) Smart integration. IEEE Power and Energy Magazine 6(6):71-79

23. Zhu Z, Lambotharan S, Chin WH, Fan Z (2012) Overview of demand management in smart grid and enabling wireless communication technologies. IEEE Wirel Commun 19(3):48-56 